\title{
A NOTE ON THE SPACES $\theta_{m}$ AND $\theta_{m}^{\prime}$
}

\author{
CARLOS BOSCH* - JAN KUCERA*" \\ *Instituto de Matemáticas \\ México, D.F. 04510 MEXICO \\ **Department of Pure and Applied Mathematics \\ Washington State University \\ Pullman, WA 99164-2930 U.S.A.
}

ABSTRACT. The spaces $\theta_{M}$ and $\theta_{C}^{\prime}$ of multiplication and convolution operators on temperate distributions, together with their strong duals $O_{M}^{\prime}$ and $O_{C}$, are Montel and distinguished.

KEY WORDS AND PHRASES. Temperate distribution, multiplication and convolution operators, inductive and projective limits, Montel space, distinguished space

1980 MATHEMATICS SUBJECT CLASSIFICATION CODE. Primary 46F10 Secondary $46 A 09$

Let $\mathbf{8}$, resp. $\mathbb{S}^{\prime}$, be the space of rapidly decreasing functions, resp. temperate distributions, on $\mathbb{R}^{n}$. Then $O_{M}$ is the space of all functions $\mathrm{f} \in C^{\infty}$ for which the map $\varphi \mapsto \varphi D^{\alpha} \mathbf{f}: \mathbb{S} \rightarrow \mathbb{S}$ is continuous for each $\alpha \in \mathbb{N}^{n}$. The topology of $O_{M}$ is generated by a family of seminorms $f \mapsto \max \left\{\left|\varphi(x) D^{\alpha} f(x)\right| ; x \in \mathbb{R}^{n}\right\}, \varphi \in \mathbb{S}, \alpha \in \mathbb{N}^{\mathbf{n}}$. Its strong dual is denoted by $0_{M}^{\prime}$.

For each $q \in \mathbb{N}$ the space

$$
\mathrm{L}_{\mathrm{q}}=\left\{\mathbf{f}: \mathbb{R}^{\mathrm{n}} \rightarrow \phi ;\|\mathbf{f}\|_{\mathrm{q}}^{2}=\sum_{|\alpha+\beta| \leq q} \int_{\mathbb{R}^{\mathrm{n}}}\left|\mathbf{x}^{\alpha} \mathrm{D}^{\beta} \mathbf{f}(\mathbf{x})\right|^{2} \mathrm{dx}<\infty\right\}
$$

is Hilbert. If we denote its dual by $L_{-q}$ we have $\mathbb{8}=\operatorname{proj} \underset{q \rightarrow \infty}{\lim } L_{q}$ and $\mathscr{S}^{\prime}=\underset{q \rightarrow \infty}{\operatorname{ind}} \lim _{\mathrm{q} \rightarrow \mathrm{q}^{\circ}}$

Put $W(x)=\left(1+|x|^{2}\right)^{\frac{1}{2}}, x \in \mathbb{R}^{n}$. Then for each integer $k$ (positive or negative) the map $T_{k}: f \rightarrow W^{k} f: \mathbb{S}^{\prime} \rightarrow \mathbb{S}^{\prime}$ is bijective. We denote by $W^{k} L_{m}$, $k, m \in z$, the image of $L_{m}$ under $T_{k}$ and provide it with the topology which makes $T_{k}: L_{m} \rightarrow W^{k} L_{m}$ a topological isomorphism.

Let $0_{q}=$ ind $\lim _{\mathrm{p} \rightarrow \infty} W^{\mathrm{P}_{\mathrm{L}}}, q \in \mathbb{N}$, and $0_{-q}$ be its strong dual. It is proved in [4] that $0_{-q}=\operatorname{proj} \lim _{\mathrm{p} \rightarrow \infty} \mathrm{W}^{-\mathrm{P}_{\mathrm{L}}}{ }_{-\mathrm{q}}$. Also, $O_{\mathrm{M}}=\underset{\mathrm{q} \rightarrow \infty}{\operatorname{proj} \lim 0_{\mathrm{q}}}$ and $O_{M}^{\prime}=$ ind $\lim _{q \rightarrow \infty} O_{-q}$, see $\left[\begin{array}{lll}3 & \& & 5\end{array}\right]$. 
PROPOSITION 1. The spaces $O_{M}$ and $\theta_{M}^{\prime}$ are Montel.

PROOF. First we prove that $O_{M}^{\prime}$ is Montel. It is ultrabornological, [5; Th. 4] and barreled [1;3-15, Ex. 9]. Hence it is infrabarreled.

Further $O_{M}^{\prime}$ is complete and Schwartz, [5; Ths. $\left.2 \& .3\right]$ and therefore it is semi-Montel, [1;3-15, Prop. 4], [6; II, 54, No. 4, Th. 16]. As infrabarreled semi-Montel space, it is Montel.

$O_{M}$ is Montel as a strong dual of the reflexive space $O_{M}^{\prime},[5$, Th. 1$]$, [1; 3-9, Prop. 9].

PROPOSITION 2. The spaces $O_{M}$ and $O_{M}^{\prime}$ are distinguished.

PROOF. Both $\theta_{M}$ and $O_{M}^{\prime}$ are ultrabornological and reflexive, [5; Ths. $1 \& 3]$. Hence they are strongly ultrabornological and strongly barreled, [1;3-15, Ex. 9]. By $[1 ; 3-16$, Prop. 1], they are distinguished. Let $\theta_{c}$ be the strong dual of the space $\theta_{C}^{\prime}$ of convolution operators on

$\boldsymbol{S}^{\prime}$. Then Fourier transformations $F: O_{M} \rightarrow O_{C}^{\prime}$ and $F: O_{M}^{\prime} \rightarrow O_{C}$ are topological isomorphisms and we have

COROLLARY. The spaces $O_{C}$ and $O_{C}^{\prime}$ are both Montel and distinguished.

\section{REFERENCES}

[1] HORVATH, J. Topological Vector Spaces and Distributions, Addison Wesley, Reading 1966.

[2] KUCERA, J. On Multipliers of Temperate Distributions, Czech. Math. J. 21, 96 (1971), 610-618.

[3] KUCERA, J., MCKENNON, K. Certain Topologies on the Space of Temperate Distributions and its Multipliers, Indiana Univ. Math. J. 24(8), (1975), 773-775.

[4] KUCERA, J., MCKENNON, K. The Topology of Certain Spaces of Multipliers of Temperate Distributions, Rocky Mountain J. of Math. $\underline{7}$ (2) (1977) 377-383.

[5] KUCERA, J. The Spaces $\theta_{M}$ and $\theta_{C}$ are ultrabornological, a new proof. Internat. J. Math. ${ }^{M}$ Math. Sci. $8(4)(1985), 813-816$.

[6] GROTHENDIECK, A., Produits Tensoriels Topologiques et Espaces Nucléaires, Mem. Am. Math. Soc. 16, (1955). 


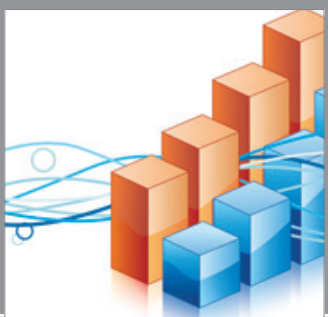

Advances in

Operations Research

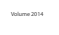

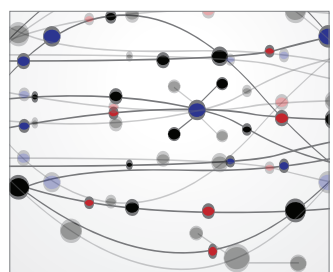

\section{The Scientific} World Journal
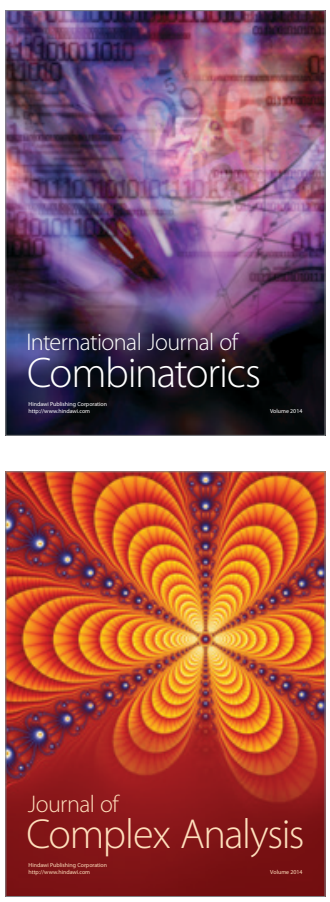

International Journal of

Mathematics and

Mathematical

Sciences
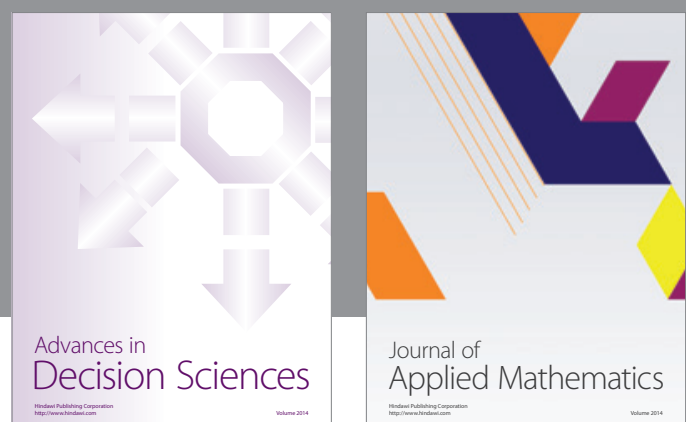

Journal of

Applied Mathematics
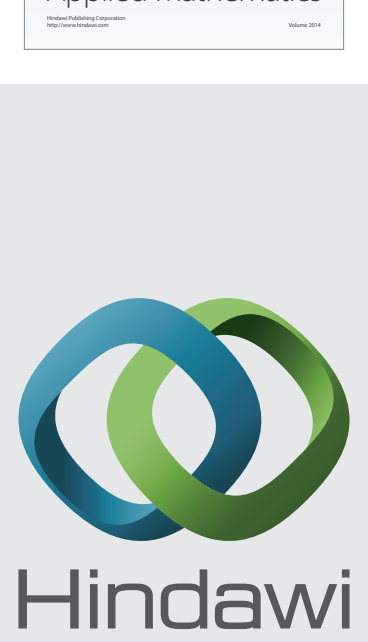

Submit your manuscripts at http://www.hindawi.com
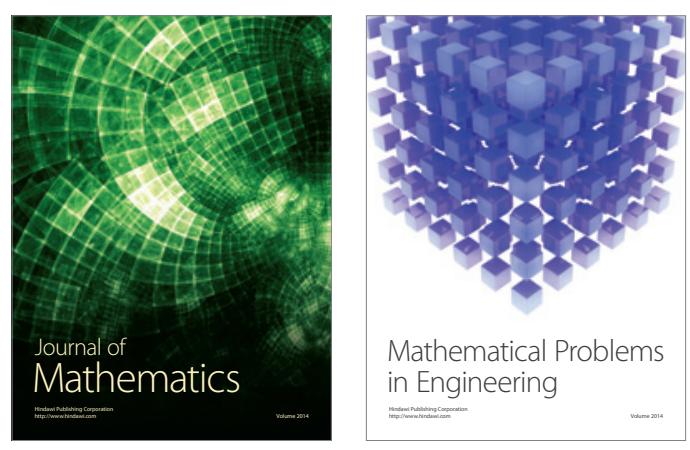

Mathematical Problems in Engineering
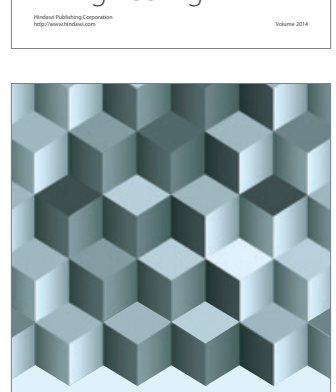

Journal of

Function Spaces
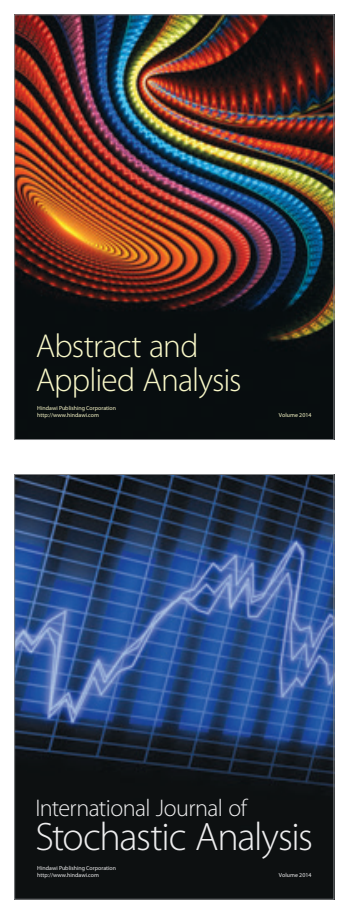

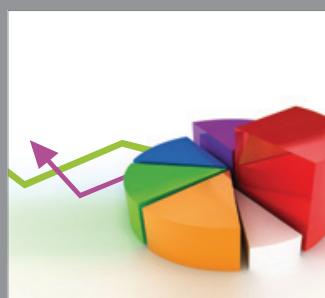

ournal of

Probability and Statistics

Promensencen
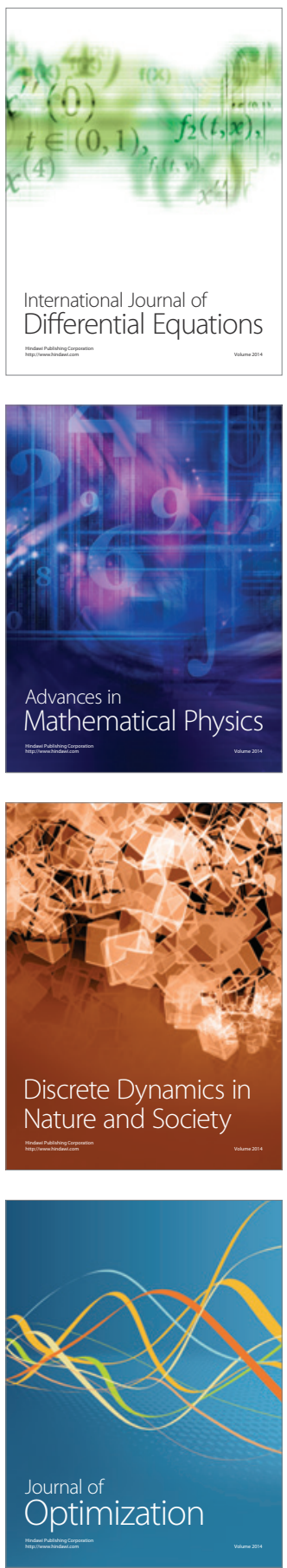